\title{
Status of Root and Foliar Fungal Diseases of Pulses at Different Agro-Climatic Zones of Uttar Pradesh, India
}

\author{
Shubha Trivedi $^{1 *}$, Mukesh Srivastava ${ }^{1}$, A.K. Srivastava ${ }^{2}$, Ved Ratan ${ }^{1}$, Mo. Shahid ${ }^{1}$, \\ Anuradha Singh ${ }^{1}$, Sonika Pandey ${ }^{1}$, Supriya Dixit ${ }^{1}$ and Y.K. Srivastava ${ }^{1}$ \\ ${ }^{1}$ Bio-control Lab, Department of Plant Pathology, ${ }^{2}$ Department of Agronomy, C. S. Azad \\ University of Agriculture and Technology, Kanpur (U.P.), India \\ *Corresponding author
}

\section{A B S T R A C T}

Pulses are high value crops and generally greater attention is paid to protect them against diseases caused by microbial pathogens after harvest during storage. An extensive field

Keywords

Survey, Pulses, Fungal

diseases,

Phytopathogens,

Bioagents,

Trichoderma sp.

Article Info

Accepted:

04 September 2017

Available Online:

10 November 2017 survey was conducted in 203 Blocks of 31 districts comprising three agro-climatic zones of Uttar Pradesh during 2011-12 and 2012-13 to find out the status of soil borne pathogens and bioagents in major pulse growing areas. In case of phytopathogens, Fusarium sp., Rhizoctonia sp., Sclerotium rolfsii, Sclerotinia sclerotiorum, Phytophthora sp., Pythium sp., Colletotrichum sp, Aschochyta rabei, Alternaria sp., Pestalotiopsis sp. were found as major disease causing agents in all 3 agro-climatic zones of Uttar Pradesh. Bioagents like Trichoderma viride and Trichoderma harzianum were prevalent in most part of the survey area of Uttar Pradesh. Other bioagents in terms of prevalence were T. atroviride, T. ressei, T. asperellum, T. virens, T. longibrachiatum, T. koningii, T. citrinoviride, T. koningiopsis, T. aggressivum, T. aureoviride, $T$. erinaceum, $T$. pubscens, $T$. saturnisporum, $T$. tomentosum, T. minutisporum and T. spirale. Based on the survey, it was found that majority of soil borne pathogens are responsible for low yield of the pulse crops. Interestingly, it was noted that the fields having much prevalence of bioagents showed low infestation of wilt/ root rot diseases.

\section{Introduction}

Pulses play a major role in nutritional security for the people taking cereal based diet. Beside their dietary value and nitrogen fixing ability, pulses also play an important role in sustaining intensive agriculture by improving physical, chemical and biological properties of soil and are considered excellent crop for diversification of cereal based cropping system. India is the largest producer and consumer of pulses, it accounts for 33 percent of the world area and 22 percent of the world production of pulse. About 90 percent of the global pigeonpea, 65 percent of chickpea and 37 percent of lentil area falls in India, corresponding to 93 percent, 68 percent and 32 percent of the global production, respectively (FAOSTAT, 2014). Pulse crop are grown across the country with the highest share coming from Madhya Pradesh (24 percent), Uttar Pradesh (16 percent), Maharashtra (14 percent), Andhra Pradesh (10 percent), Karnataka (7 percent) and Rajasthan (6 percent), which together share about 77 percent of the total pulse production, 
while the remaining 23 percent is contributed by Gujarat, Chhattisgarh, Bihar, Orissa and Jharkhand

Uttar Pradesh has large area under pulses (2522000 ha). However, the production (2197.2 th tonnes) of pulses (with an average of $871 \mathrm{~kg} / \mathrm{ha}$ yield) in this region does not commensurate with the acreage. The reason for this situation is invasion of pulses by several soil borne pathogens. No systematic information is available on the status of soil borne pathogens as well as bioagents present in this area.

Bioagent such as Trichoderma sp. is most commonly used biological control agent and have long been known as effective antagonistic against plant pathogenic fungi (Chet et al., 1981; Papavizas, 1985; Chet, 1987; Kumar and Mukharjii, 1996). Trichoderma as a potent fungal biocontrol agent against a range of plant pathogens has attracted considerable scientific attention during last few decades (Tewari and Mukhopadhyay, 2001; Rini and Sulochana, 2007). The antifungal action of Trichoderma spp. is well documented against number of foliar and soil borne fungi like. Fusarium sp., Pythium sp., Rhizoctonia solani, Sclerotium rolfsii, in vegetables, field, fruit and industrial crops (Tran, 1998; Ngo et al., 2006).

Therefore the present investigations were made to find out the actual situation of soil borne pathogens as well as bioagents present in Uttar Pradesh.

\section{Materials and Methods}

\section{Survey}

An extensive survey was conducted in 203 Blocks of 31 districts comprising three agroclimatic zones of Uttar Pradesh during 2011$12 \& 2012-13$ to observe the disease incidence in major pulse growing areas. In each district, randomly 15 soil samples from $5-6 \mathrm{~cm}$ depth were collected from the fields of villages adjoining to Block Headquarters. Each soil sample kept in parchment paper bags covered with polythene bags properly labeled with the name of district, block, village, name of the farmer, crop and date of the collection were brought to Bio-control Lab of the department of Plant Pathology, C.S. Azad University of Agriculture and Technology, Kanpur.

\section{Isolation, purification and identification of soil borne fungal pathogens}

Isolation was made from $1 \mathrm{~g}$ soil dissolved in $10 \mathrm{ml}$ sterilized distilled water. Five fold serial dilutions were made from each soil sample and $0.5 \mathrm{ml}$ of diluted sample was then poured on already prepared PDA medium in Petri plates. All Petri plates were incubated at $25+1^{\circ} \mathrm{C}$ for 96 hours. Morphologically different colonies appeared on the plate were purified and observed under steriobincular microscope.

The colonies appeared as fungi like Fusarium, Rhizoctonia, Sclerotium, Sclerotinia, Phytophthora, Pythium, Colletotrichum, Ascochyta, Alternaria, Pestalotiopsis were separated. Hyphal tips of different fungi were picked up and transferred to another PDA plates. Purified fungi were identified based on their microscopic and morphological characters (Gilman, 1957; Barnett et al., 1972; Nelson et al., 1983). For confirmation at species level, the samples were sent to ITCC, New Delhi.

\section{Isolation, purification and identification of} fungal bioagents

From the rhizospheric soil samples, Trichoderma species were isolated by dilution plate technique (Johnson, 1957). Trichoderma 
selective medium (TSM) was used for isolation (Elad et al., 1991). After few days of incubation, colonies appear in varying densities, depending upon the amount of dilution from the original material. The fungal colonies developed in the plates were sub cultured and purified on TSM slants. For confirmation at species level, the samples were sent to ITCC, New Delhi.

\section{Prevalence of pathogens and bioagents}

Number of colonies for each pathogen/ bioagent developed from the soil samples was counted and percent recovery of each pathogen and bioagent was worked out. From each soil sample, 10 isolations were made. Based on the frequency of the pathogen/ bioagent appeared on PDA plates, number of pathogen and bioagents was counted and prevalence percentage was determined. The observations recorded are summarized in Tables 1 and 2.

\section{Statistical analysis}

All experiments were set up in a complete randomized design. One-way ANOVA was used to analyze differences between applied treatments. A general linear model option of the analysis system SAS (SAS Institute Inc., 1996) was used to perform the ANOVA. Region-wise prevalence correlation coefficient of bioagents vs. pathogens was also worked out (Table 3).

\section{Results and Discussion}

Prevalence of phytopathogens isolated from soil samples of different places of Uttar Pradesh

It is evident from Table 1 that, out of 34 Blocks of six districts surveyed in Bundelkhand Region, five pathogens viz., Fusarium, Rhizoctonia, Sclerotium rolfsii,
Sclerotinia sclerotiorum, Phytophthora and Ascochyta rabei were isolated. Four types of pathogens were isolated from eight Blocks of Banda, seven Blocks of Hamirpur and four Blocks of Lalitpur districts; three types of pathogens were isolated from five Blocks of Jalaun and six blocks of Jhansi and only two types of pathogens could be isolated from four Blocks of Mahoba. In general, 26.85 per cent pathogens were isolated from the entire zone.

Statistically, Fusarium sp. given high response in Banda, Hamirpur, Jhansi, Mahoba and Lalitpur followed by Rhizoctonia sp. Sclerotinia sclerotiorum and Phytophthora sp. were statistically at par to each other and given same response in Bundelkhand region.

Out of 76 Blocks of eleven districts surveyed in Central Region, eleven pathogens viz., Fusarium, Rhizoctonia, Sclerotium rolfsii, Sclerotinia sclerotiorum, Phytophthora, Pythium, Colletotrichum, Ascochyta rabei, Alternaria, and Pestalotiopsis were isolated.

Maximum nine type of pathogens were isolated from six Blocks of Fatehpur; eight type of pathogens were isolated from eight Blocks of Etawah, seven Blocks of Kanpur Nagar, eight Blocks of Raebareli and seven Blocks of Unnao; seven type of pathogens were isolated from five Blocks of Hardoi, five Blocks of Kaushambi and seven Blocks of Lucknow and six types of pathogens were isolated from seven Blocks of Auraiya, six Blocks of Kanpur Dehat and ten Blocks of Sitapur. In general, 26.19 per cent pathogens were isolated from the entire zone.

Statistically, Fusarium sp. given high response in Fatehpur, Raibareilly, Kanpur Nagar and Kanpur Dehat. Rhizoctonia sp. and Alternaria sp. given same response in Raibareilly, Kaushambi and Fatehpur. Phytophthora sp., Pythium sp., $S$. 
sclerotiorum, Sclerotium rolfsii and Ascochyta sp. were statistically at par with each other in Fatehpur, Kaushambi, Raebareli and Unnao.

Out of 93 Blocks of fourteen districts surveyed in Eastern Region, ten pathogens viz., Fusarium, Rhizoctonia, Sclerotium rolfsii, Sclerotinia sclerotiorum, Phytophthora, Pythium, Colletotrichum, Ascochyta rabei, Alternaria and sterility mosaic virus were isolated.

Maximum eight type of pathogens were isolated from six Blocks of Faizabad; seven type of pathogens were isolated from nine Blocks each of Allahabad, Azamgarh, Jaunpur and Sultanpur, eight Blocks of Barabanki, six Blocks of Sonbhadra and five Blocks each of Gonda and Sant Ravidas Nagar; six type of pathogens were isolated from five Blocks of Bahraich; five type of pathogens were isolated from six Blocks each of Mirzapur and Varanasi and five Blocks of Ghazipur and only four type of pathogens were isolated from five Blocks of Pratapgarh.

In general, 25.24 per cent pathogens were isolated from the entire zone (Figs. 1, $2 \& 3$ ).

Statistically, Fusarium sp. given high response in Barabanki, Gonda, Sonbhadra followed by Rhizoctonia sp., S. sclerotiorum, Sclerotium rolfsii, Phytophthora sp., Alternaria sp. and Pythium sp. have also given some incidence in Sonbhadra, Barabanki, Azamgarh, Gonda, Faizabad and Varanasi.

The isolated fungi were previously recorded as the main causal of root diseases of surveyed pulse and vegetable crops by Jones (2001), Jude and Richard (2001), Elizabeth et al., (2001), Pernezny et al., (2003), Momol and Pernezny (2005), Trivedi and Gurha (2006), Gurha et al., (2007).
Prevalence of bioagents isolated from soil samples of different places of Uttar Pradesh

It is evident from Table 2 that a total of nine Trichoderma species viz; $T$. harzianum, $T$. viride, T. virens, T. asperellum, T. koningii, $T$. ressei, $T$. aureoviride, $T$. longibrachiatum and $T$. atroviride could be isolated from soil samples of Bundelkhand region of U. P. 7 species of bioagents from 7 Blocks of Hamirpur and 5 Blocks of Jalaun; 6 species of bioagents from 8 Blocks of Banda; 5 species of bioagents from 5 Blocks of Jhansi; 4 species of bioagents from 4 Blocks of Mahoba and 3 species of bioagents from 4 Blocks of Lalitpur could be isolated. In general, 20.61 per cent bioagents were isolated from this zone.

T. viride has given most effective response to the pathogens while, $T$. harzianum, $T$. asperellum and $T$. koningii were recorded statistically at par given same response over pathogens in Hamirpur, Jhansi, Lalitpur and Mahoba districts of Bundelkhand region.

In addition, fifteen Trichoderma species viz; $T$. harzianum, $T$. viride, $T$. virens, $T$. asperellum, $T$. atroviride, T. koningii, $T$. ressei, T. citrinoviride, $T$. spirale, $T$. erinaceum, T. pubscens, T. tomentosum, $T$. minutisporum, $T$. koningiopsis and $T$. longibrachiatum could be isolated from soil samples of Central region of U.P. 10 species of bioagents from 7 Blocks of Kanpur Nagar, 8 species of bioagents from 8 Blocks of Etawah and 6 Blocks of Kanpur Dehat; 7 species of bioagents from 10 Blocks of Sitapur; 6 species of bioagents from 8 Blocks of Raebareli, 7 Blocks of Auraiya, 6 Blocks of Fatehpur, 5 Blocks each of Hardoi and Kaushambi and 5 species of bioagents from 7 Blocks each of Lucknow and Unnao. In general, 20.89 per cent bioagents were isolated from this zone. 


\section{Int.J.Curr.Microbiol.App.Sci (2017) 6(11): 152-165}

Table.1 Prevalence of phytopathogens isolated from soil samples of different places of Uttar Pradesh

\begin{tabular}{|c|c|c|c|c|c|c|c|c|c|c|c|c|}
\hline District & \multicolumn{12}{|c|}{ Pathogens (\%) } \\
\hline $\begin{array}{c}\text { Bundelkhand } \\
\text { Region }\end{array}$ & \begin{tabular}{|c|} 
Fusarium \\
sp.
\end{tabular} & \begin{tabular}{|c|} 
Rhizoctonia \\
sp.
\end{tabular} & $\begin{array}{c}\text { Sclerotium } \\
\text { rolfsii }\end{array}$ & \begin{tabular}{|c|}
$S$. \\
sclerotiorum \\
\end{tabular} & $\begin{array}{l}\text { Phytophtho } \\
\text { ra sp. }\end{array}$ & $\begin{array}{c}\text { Pythium } \\
\text { sp. }\end{array}$ & $\begin{array}{c}\text { Colletotrichum } \\
\text { sp }\end{array}$ & $\begin{array}{c}\text { Aschochyt } \\
\text { a rabei }\end{array}$ & $\begin{array}{l}\text { Alterna } \\
\text { ria sp. }\end{array}$ & $\begin{array}{c}\text { Pestalotiopsis } \\
\text { sp. }\end{array}$ & Mean & SD \\
\hline Banda & 17.5 & 3.75 & \begin{tabular}{|l|l|}
3.75 \\
\end{tabular} & 0.0 & 2.5 & 0.0 & 0.0 & 0.0 & 0.0 & 0.0 & 2.5 & 5.2 \\
\hline Hamirpur & 12.8 & 10.0 & 3.3 & 0.0 & 1.4 & 0.0 & 0.0 & 0.0 & 0.0 & 0.0 & 2.5 & 4.6 \\
\hline Jalaun & 12.0 & 6.0 & 0.0 & 0.0 & 4.0 & 0.0 & 0.0 & 0.0 & 0.0 & 0.0 & 2.0 & 3.9 \\
\hline Jhansi & 16.6 & 5.0 & 5.0 & 0.0 & 0.0 & 1.6 & 0.0 & 0.0 & 0.0 & 0.0 & 2.6 & 5.1 \\
\hline Lalitpur & 12.5 & 7.5 & 5.0 & 0.0 & 0.0 & 0.0 & 0.0 & 2.5 & 0.0 & 0.0 & 2.5 & 4.2 \\
\hline Mahoba & 17.5 & 12.5 & 0.0 & 0.0 & 0.0 & 0.0 & 0.0 & 0.0 & 0.0 & 0.0 & 2.7 & 6.2 \\
\hline SE $(M) \pm$ & \multicolumn{12}{|c|}{1.04} \\
\hline CD (5\%) & & & & & & 06 & & & & & & \\
\hline \multicolumn{13}{|c|}{ Central Region } \\
\hline Auraiya & 12.8 & 2.8 & 0.0 & 1.4 & 0.0 & 1.4 & 1.4 & 0.0 & 4.2 & 0.0 & 2.2 & 3.8 \\
\hline Etawah & 11.2 & 2.5 & 1.2 & 1.2 & 1.2 & 1.2 & 1.2 & 0.0 & 2.5 & 0.0 & 2.0 & 3.2 \\
\hline Fatehpur & 13.3 & 3.3 & 1.6 & 1.6 & 1.6 & 3.3 & 1.6 & 1.6 & 3.3 & 0.0 & 2.8 & 3.7 \\
\hline Hardoi & 8.0 & 4.0 & 2.0 & 2.0 & 2.0 & 2.0 & 0.0 & 0.0 & 0.0 & 0.0 & 2.2 & 2.4 \\
\hline Kanpur Dehat & 10.0 & 5.0 & 1.6 & 1.6 & 0.0 & 0.0 & 0.0 & 3.3 & 3.3 & 0.0 & 2.3 & 3.1 \\
\hline Kanpur Nagar & 12.8 & 2.8 & 1.4 & 1.4 & 1.4 & 0.0 & 1.4 & 1.4 & 0.0 & 4.2 & 2.4 & 3.7 \\
\hline Kaushambi & 12.0 & 4.0 & 4.0 & 2.0 & 0.0 & 2.0 & 2.0 & 0.0 & 4.0 & 0.0 & 2.7 & 3.5 \\
\hline Lucknow & 8.5 & 2.8 & 1.4 & 0.0 & 2.8 & 1.4 & 0.0 & 0.0 & 1.4 & 1.4 & 1.8 & 2.5 \\
\hline Raebareli & 11.2 & 6.2 & 2.5 & 1.2 & 6.2 & 1.2 & 1.2 & 0.0 & 2.5 & 0.0 & 2.9 & 3.5 \\
\hline Sitapur & 11.0 & 4.0 & 3.0 & 0.0 & 0.0 & 2.0 & 0.0 & 3.0 & 4.0 & 0.0 & 2.5 & 3.3 \\
\hline Unnao & 10.0 & 2.8 & 0.0 & 1.4 & 1.4 & 1.4 & 1.4 & 2.8 & 4.2 & 0.0 & 2.3 & 2.9 \\
\hline SE $(M) \pm$ & \multicolumn{12}{|c|}{0.41} \\
\hline CD (5\%) & & & & & & 1.15 & & & & & & \\
\hline \multicolumn{13}{|c|}{ Eastern Region } \\
\hline Allahabad & 12.2 & 3.3 & 1.1 & 1.1 & 2.2 & 1.1 & 0.0 & 0.0 & 1.1 & 0.0 & 2.0 & 3.5 \\
\hline Azamgarh & 11.1 & 5.5 & 0.0 & 2.2 & 1.1 & 1.1 & 1.1 & 0.0 & 5.5 & 0.0 & 2.5 & 3.5 \\
\hline Bahraich & 10.0 & 2.0 & 2.0 & 4.0 & 0.0 & 2.0 & 4.0 & 0.0 & 0.0 & 0.0 & 2.2 & 3.0 \\
\hline Barabanki & 17.5 & 3.75 & 2.5 & 2.5 & 2.5 & 1.2 & 0.0 & 0.0 & 1.2 & 0.0 & 2.8 & 5.0 \\
\hline Faizabad & 11.6 & 1.6 & 1.6 & 3.3 & 1.6 & 0.0 & 1.6 & 0.0 & 1.6 & 0.0 & 2.4 & 3.3 \\
\hline Ghazipur & 10.0 & 8.0 & 2.0 & 2.0 & 0.0 & 2.0 & 0.0 & 0.0 & 0.0 & 0.0 & 2.2 & 3.5 \\
\hline Gonda & 10.0 & 4.0 & 4.0 & 0.0 & 2.0 & 2.0 & 0.0 & 0.0 & 2.0 & 0.0 & 2.5 & 3.0 \\
\hline Jaunpur & 15.5 & 2.2 & 1.1 & 1.1 & 2.2 & 2.2 & 0.0 & 0.0 & 2.2 & 0.0 & 2.4 & 4.5 \\
\hline Mirzapur & 11.6 & 5.0 & 1.6 & 0.0 & 3.3 & 1.6 & 0.0 & 0.0 & 0.0 & 0.0 & 2.1 & 3.6 \\
\hline Pratapgarh & 10.0 & 8.0 & 0.0 & 4.0 & 0.0 & 0.0 & 2.0 & 0.0 & 0.0 & 0.0 & 2.2 & 3.6 \\
\hline $\begin{array}{l}\text { Sant Ravidas } \\
\text { Nagar }\end{array}$ & 8.0 & 4.0 & 2.0 & 0.0 & 2.0 & 2.0 & 2.0 & 0.0 & 2.0 & 0.0 & 2.0 & 2.4 \\
\hline Sonbhadra & 13.3 & 5.0 & 3.3 & 3.3 & 0.0 & 1.6 & 1.6 & 0.0 & 1.6 & 0.0 & 2.7 & 3.9 \\
\hline Sultanpur & 6.6 & 4.4 & 2.2 & 3.3 & 2.2 & 0.0 & 0.0 & 0.0 & 2.2 & 0.0 & 2.0 & 2.1 \\
\hline Varanasi & 10.0 & 8.3 & 0.0 & 0.0 & 1.6 & 0.0 & 0.0 & 1.6 & 1.6 & 0.0 & 2.1 & 3.6 \\
\hline SE $(M) \pm$ & \multicolumn{12}{|c|}{0.46} \\
\hline CD (5\%) & & & & & & 1.28 & & & & & & \\
\hline
\end{tabular}


Table.2 Prevalence of bioagents isolated from soil samples of different places of Uttar Pradesh

\begin{tabular}{|c|c|c|c|c|c|c|c|c|c|c|c|c|c|c|c|c|c|c|c|c|c|}
\hline District & \multicolumn{21}{|c|}{ Bio-agents (\%) } \\
\hline $\begin{array}{c}\text { Bundelkhand } \\
\text { Region }\end{array}$ & $\begin{array}{c}T . \\
\text { harzian- } \\
\text { um }\end{array}$ & $\begin{array}{c}T . \\
\text { viride }\end{array}$ & $\begin{array}{c}T . \\
\text { virens }\end{array}$ & $\begin{array}{c}T . \\
\text { aspere- } \\
\text { llum }\end{array}$ & $\begin{array}{c}T . \\
\text { atro- } \\
\text { viride }\end{array}$ & $\begin{array}{c}T . \\
\text { longibra- } \\
\text { chiatum }\end{array}$ & $\begin{array}{c}T . \\
\text { koningii }\end{array}$ & $\begin{array}{c}T . \\
\text { reesei }\end{array}$ & $\begin{array}{c}T . \\
\text { aggres- } \\
\text { sium }\end{array}$ & $\begin{array}{c}T . \\
\text { citrino- } \\
\text { viride }\end{array}$ & $\begin{array}{c}T . \\
\text { spirale }\end{array}$ & $\begin{array}{c}T . \\
\text { erinac- } \\
\text { eum }\end{array}$ & $\begin{array}{c}T . \\
\text { pubscens }\end{array}$ & $\begin{array}{c}T . \\
\text { tomen- } \\
\text { tosum }\end{array}$ & $\begin{array}{c}T . \\
\text { minutis- } \\
\text { porum }\end{array}$ & $\begin{array}{c}T . \\
\text { koningio- } \\
\text { psis }\end{array}$ & $\begin{array}{c}T . \\
\text { saturn- } \\
\text { isporum }\end{array}$ & $\begin{array}{c}T . \\
\text { aureov- } \\
\text { iride }\end{array}$ & $\begin{array}{c}P . \\
\text { fluoresc- } \\
\text { ens }\end{array}$ & Mean & SD \\
\hline Banda & 3.75 & 8.75 & 2.5 & 1.2 & 0.0 & 1.2 & 0.0 & 1.2 & 0.0 & 0.0 & 0.0 & 0.0 & 0.0 & 0.0 & 0.0 & 0.0 & 0.0 & 0.0 & 0.0 & 0.2 & 0.5 \\
\hline Hamirpur & 5.7 & 10 & 1.4 & 5.7 & 0.0 & 0.0 & 1.4 & 1.4 & 0.0 & 0.0 & 0.0 & 0.0 & 0.0 & 0.0 & 0.0 & 0.0 & 0.0 & 0.0 & 0.0 & 0.5 & 1.5 \\
\hline Jalaun & 6.0 & 4.0 & 0.0 & 2.0 & 2.0 & 2.0 & 0.0 & 2.0 & 0.0 & 0.0 & 0.0 & 0.0 & 0.0 & 0.0 & 0.0 & 0.0 & 0.0 & 0.0 & 0.0 & 0.5 & 0.9 \\
\hline Jhansi & 1.6 & 10 & 0.0 & 3.3 & 0.0 & 0.0 & 1.6 & 0.0 & 0.0 & 0.0 & 0.0 & 0.0 & 0.0 & 0.0 & 0.0 & 0.0 & 0.0 & 5.0 & 0.0 & 0.6 & 1.5 \\
\hline Lalitpur & 0.0 & 15 & 0.0 & 2.5 & 2.5 & 0.0 & 0.0 & 0.0 & 0.0 & 0.0 & 0.0 & 0.0 & 0.0 & 0.0 & 0.0 & 0.0 & 0.0 & 0.0 & 0.0 & 0.3 & 0.9 \\
\hline Mahoba & 5.0 & 10 & 0.0 & 2.5 & 0.0 & 0.0 & 2.5 & 0.0 & 0.0 & 0.0 & 0.0 & 0.0 & 0.0 & 0.0 & 0.0 & 0.0 & 0.0 & 0.0 & 0.0 & 0.3 & 0.9 \\
\hline SE $(M) \pm$ & \multicolumn{21}{|c|}{1.07} \\
\hline CD (5\%) & & & & & & & & & & 3. & & & & & & & & & & & \\
\hline \multicolumn{22}{|c|}{$\begin{array}{l}3.22 \\
\text { Central region }\end{array}$} \\
\hline Auraiya & 7.1 & 5.7 & 1.4 & 0.0 & 5.7 & 0.0 & 1.4 & 2.8 & 0.0 & 0.0 & 0.0 & 0.0 & 0.0 & 0.0 & 0.0 & 0.0 & 0.0 & 0.0 & 0.0 & 0.6 & 1.6 \\
\hline Etawah & 3.75 & 2.5 & 1.25 & 1.25 & 1.25 & 0.0 & 3.75 & 3.75 & 0.0 & 0.0 & 3.75 & 0.0 & 0.0 & 0.0 & 0.0 & 0.0 & 0.0 & 0.0 & 0.0 & 0.9 & 1.5 \\
\hline Fatehpur & 1.6 & 6.6 & 3.3 & 1.6 & 0.0 & 0.0 & 0.0 & 3.3 & 0.0 & 0.0 & 0.0 & 0.0 & 0.0 & 0.0 & 0.0 & 0.0 & 0.0 & 0.0 & 0.0 & 0.3 & 0.9 \\
\hline Hardoi & 6.0 & 8.0 & 2.0 & 2.0 & 2.0 & 0.0 & 0.0 & 2.0 & 0.0 & 0.0 & 0.0 & 0.0 & 0.0 & 0.0 & 0.0 & 0.0 & 0.0 & 0.0 & 0.0 & 0.4 & 0.8 \\
\hline Kanpur Dehat & 0.0 & 5.0 & 0.0 & 1.6 & 3.3 & 1.6 & 3.0 & 3.0 & 0.0 & 0.0 & 0.0 & 0.0 & 0.0 & 3.0 & 3.0 & 0.0 & 0.0 & 0.0 & 0.0 & 1.2 & 1.4 \\
\hline Kanpur Nagar & 4.2 & 8.5 & 5.7 & 2.8 & 0.0 & 1.4 & 0.0 & 2.8 & 0.0 & 5.7 & 5.7 & 4.2 & 4.2 & 0.0 & 0.0 & 0.0 & 0.0 & 0.0 & 0.0 & 1.7 & 2.2 \\
\hline Kaushambi & 0.0 & 4.0 & 2.0 & 2.0 & 4.0 & 0.0 & 2.0 & 2.0 & 0.0 & 0.0 & 0.0 & 0.0 & 0.0 & 0.0 & 0.0 & 0.0 & 0.0 & 0.0 & 0.0 & 0.6 & 1.2 \\
\hline Lucknow & 2.8 & 1.4 & 0.0 & 0.0 & 1.4 & 1.4 & 0.0 & 0.0 & 0.0 & 0.0 & 0.0 & 0.0 & 0.0 & 0.0 & 0.0 & 0.0 & 0.0 & 0.0 & 0.0 & 0.2 & 0.5 \\
\hline Raebareli & 2.5 & 7.5 & 0.0 & 0.0 & 1.2 & 0.0 & 1.2 & 1.2 & 0.0 & 0.0 & 0.0 & 0.0 & 0.0 & 0.0 & 0.0 & 6.25 & 0.0 & 0.0 & 0.0 & 0.6 & 1.6 \\
\hline Sitapur & 4.0 & 4.0 & 3.0 & 2.0 & 2.0 & 0.0 & 2.0 & 0.0 & 0.0 & 2.0 & 0.0 & 0.0 & 0.0 & 0.0 & 0.0 & 0.0 & 0.0 & 0.0 & 0.0 & 0.5 & 0.9 \\
\hline Unnao & 0.0 & 5.7 & 1.4 & 0.0 & 2.8 & 1.4 & 1.4 & 2.8 & 0.0 & 0.0 & 0.0 & 0.0 & 0.0 & 0.0 & 0.0 & 0.0 & 0.0 & 0.0 & 0.0 & 0.5 & 1.0 \\
\hline $\mathrm{SE}(\mathrm{M}) \pm$ & \multicolumn{21}{|c|}{0.52} \\
\hline CD (5\%) & & & & & & & & & & 1. & 47 & & & & & & & & & & \\
\hline \multicolumn{22}{|c|}{ Region } \\
\hline Allahabad & 0.0 & 6.6 & 2.2 & 2.2 & 2.2 & 1.1 & 1.1 & 2.2 & 0.0 & 0.0 & 0.0 & 0.0 & 0.0 & 0.0 & 0.0 & 0.0 & 3.3 & 0.0 & 1.1 & 0.8 & 1.1 \\
\hline Azamgarh & 2.2 & 3.3 & 3.3 & 1.1 & 3.3 & 1.1 & 2.2 & 0.0 & 0.0 & 0.0 & 0.0 & 0.0 & 0.0 & 0.0 & 0.0 & 0.0 & 0.0 & 0.0 & 0.0 & 0.5 & 1.0 \\
\hline Bahraich & 6.0 & 6.0 & 0.0 & 2.0 & 4.0 & 2.0 & 0.0 & 0.0 & 0.0 & 0.0 & 0.0 & 0.0 & 0.0 & 0.0 & 0.0 & 0.0 & 0.0 & 0.0 & 0.0 & 0.5 & 1.2 \\
\hline Barabanki & 3.7 & 7.5 & 1.2 & 2.5 & 1.2 & 2.5 & 1.2 & 0.0 & 0.0 & 0.0 & 0.0 & 0.0 & 0.0 & 0.0 & 0.0 & 0.0 & 0.0 & 0.0 & 0.0 & 0.5 & 0.9 \\
\hline Faizabad & 5.0 & 1.6 & 0.0 & 3.3 & 3.3 & 1.6 & 0.0 & 3.3 & 0.0 & 0.0 & 0.0 & 0.0 & 0.0 & 0.0 & 0.0 & 0.0 & 0.0 & 0.0 & 0.0 & 0.7 & 1.3 \\
\hline Ghazipur & 12 & 2.0 & 4.0 & 2.0 & 0.0 & 0.0 & 0.0 & 2.0 & 0.0 & 0.0 & 0.0 & 0.0 & 0.0 & 0.0 & 0.0 & 0.0 & 0.0 & 0.0 & 0.0 & 0.3 & 0.7 \\
\hline Gonda & 0.0 & 4.0 & 4.0 & 2.0 & 2.0 & 0.0 & 2.0 & 4.0 & 0.0 & 0.0 & 0.0 & 0.0 & 0.0 & 0.0 & 0.0 & 0.0 & 0.0 & 0.0 & 0.0 & 0.6 & 1.2 \\
\hline Jaunpur & 5.5 & 7.7 & 2.2 & 1.1 & 5.5 & 0.0 & 1.1 & 1.1 & 0.0 & 0.0 & 0.0 & 0.0 & 0.0 & 0.0 & 0.0 & 0.0 & 0.0 & 0.0 & 0.0 & 0.6 & 1.4 \\
\hline Mirzapur & 5.0 & 8.3 & 3.3 & 0.0 & 3.3 & 3.3 & 3.3 & 5.0 & 0.0 & 0.0 & 0.0 & 0.0 & 0.0 & 0.0 & 0.0 & 0.0 & 0.0 & 0.0 & 0.0 & 0.9 & 1.7 \\
\hline Pratapgarh & 2.0 & 6.0 & 2.0 & 2.0 & 2.0 & 0.0 & 0.0 & 6.0 & 6.0 & 0.0 & 0.0 & 0.0 & 0.0 & 0.0 & 0.0 & 0.0 & 0.0 & 0.0 & 0.0 & 1.0 & 2.1 \\
\hline Sonbhadra & 5.0 & 3.3 & 1.6 & 3.3 & 3.3 & 1.6 & 1.6 & 1.6 & 0.0 & 0.0 & 0.0 & 0.0 & 0.0 & 0.0 & 0.0 & 0.0 & 0.0 & 0.0 & 0.0 & 0.7 & 1.2 \\
\hline SR Nagar & 4.0 & 8.0 & 0.0 & 2.0 & 4.0 & 6.0 & 0.0 & 2.0 & 0.0 & 0.0 & 0.0 & 0.0 & 0.0 & 0.0 & 0.0 & 0.0 & 0.0 & 0.0 & 0.0 & 0.9 & 1.8 \\
\hline Sultanpur & 8.8 & 5.5 & 1.1 & 0.0 & 2.2 & 2.2 & 0.0 & 1.1 & 0.0 & 0.0 & 0.0 & 0.0 & 0.0 & 0.0 & 0.0 & 0.0 & 0.0 & 0.0 & 0.0 & 0.3 & 0.8 \\
\hline Varanasi & 10 & 5.0 & 1.6 & 0.0 & 1.6 & 0.0 & 0.0 & 1.6 & 0.0 & 0.0 & 0.0 & 0.0 & 0.0 & 0.0 & 0.0 & 0.0 & 0.0 & 0.0 & 0.0 & 0.2 & 0.5 \\
\hline SE $(M) \pm$ & \multicolumn{21}{|c|}{$\mathbf{0 . 5 4}$} \\
\hline CD (5\%) & \multicolumn{21}{|c|}{1.51} \\
\hline
\end{tabular}


Table.3 Region-wise prevalence correlation coefficient

\begin{tabular}{|l|c|c|c|c|}
\hline Bundelkhand & \multicolumn{4}{|c|}{ Correlation Coefficient } \\
\hline \multirow{2}{*}{ Bioagents } & \multicolumn{4}{|c|}{ Pathogens } \\
\cline { 2 - 5 } & Fusarium sp. & Rhizoctonia sp. & S. rolfsii & 0.6150 \\
\hline T. harzianum & -0.0647 & 0.3314 & -0.7848 & -0.8277 \\
\hline T. viride & 0.0423 & 0.2359 & 0.6603 & -0.2847 \\
\hline T. asperellum & -0.3468 & 0.4609 & 0.1924 & -0.5939 \\
\hline T. koningii & 0.4762 & 0.6966 & -0.2442 & \\
\hline
\end{tabular}

\begin{tabular}{|c|c|c|c|c|c|c|c|c|c|}
\hline \multirow{3}{*}{\begin{tabular}{|r|} 
Central \\
Bioagents
\end{tabular}} & \multicolumn{9}{|c|}{ Correlation Coefficient } \\
\hline & \multicolumn{9}{|c|}{ Pathogens } \\
\hline & $\begin{array}{c}\text { Fusariu } \\
\text { m sp. }\end{array}$ & $\begin{array}{c}\text { Rhizoctonia } \\
\text { sp. }\end{array}$ & $\begin{array}{c}\text { S. } \\
\text { rolfsii }\end{array}$ & $\begin{array}{c}S . \\
\text { sclerotiorum }\end{array}$ & $\begin{array}{l}\text { Phytophthora } \\
\text { sp. }\end{array}$ & $\begin{array}{c}\text { Pythium } \\
\text { sp. }\end{array}$ & $\begin{array}{c}\text { Colletotrichum } \\
\text { sp. }\end{array}$ & $\begin{array}{c}\text { Aschochyta } \\
\text { sp. }\end{array}$ & $\begin{array}{c}\text { Alternari } \\
\quad \text { a sp. }\end{array}$ \\
\hline T. harzianum & 0.0204 & -0.2594 & -0.2535 & -0.1354 & 0.0015 & 0.0204 & -0.2033 & -0.4438 & -0.3879 \\
\hline T. viride & 0.2456 & 0.3076 & -0.0736 & 0.5595 & 0.2673 & -0.0685 & 0.2203 & 0.0686 & -0.3454 \\
\hline T. virens & 0.5247 & -0.3817 & 0.0829 & 0.1319 & -0.3059 & 0.0834 & 0.3119 & 0.1751 & -0.2889 \\
\hline T. asperellum & 0.2025 & -0.0152 & 0.4957 & 0.2760 & -0.4284 & -0.0389 & -0.0389 & 0.2252 & -0.3530 \\
\hline T. atroviride & -0.0141 & 0.0182 & -0.1219 & 0.2117 & -0.4781 & -0.0869 & 0.0694 & -0.0545 & 0.5717 \\
\hline T. koningii & 0.0616 & 0.1189 & 0.0804 & 0.0178 & -0.3559 & -0.2881 & 0.0185 & 0.2025 & 0.5209 \\
\hline T. ressei & 0.4225 & -0.2907 & -0.4481 & $0.7133^{*}$ & -0.2873 & -0.1284 & 0.5204 & 0.0530 & 0.0737 \\
\hline
\end{tabular}

\begin{tabular}{|c|c|c|c|c|c|c|c|}
\hline \multirow[b]{3}{*}{ Bioagents } & \multicolumn{7}{|c|}{ Correlation Coefficient } \\
\hline & \multicolumn{7}{|c|}{ Pathogens } \\
\hline & $\begin{array}{c}\text { Fusarium } \\
\text { sp. }\end{array}$ & $\begin{array}{c}\text { Rhizoctonia } \\
\text { sp. }\end{array}$ & S. rolfsii & S. sclerotiorum & Phytophthora sp. & $\begin{array}{c}\text { Pythium } \\
\text { sp. }\end{array}$ & Alternaria sp. \\
\hline T. harzianum & -0.2842 & 0.3532 & -0.1072 & 0.0344 & -0.1756 & -0.0939 & -0.2360 \\
\hline T. viride & 0.5031 & -0.1201 & 0.0699 & 0.0086 & 0.2517 & 0.1509 & -0.2609 \\
\hline T. virens & -0.0992 & 0.4201 & -0.0723 & $-0.5750 *$ & 0.2138 & 0.3219 & 0.1266 \\
\hline T. asperellum & 0.1718 & -0.3685 & 0.2975 & 0.2172 & -0.2656 & 0.2142 & -0.1107 \\
\hline T. atroviride & 0.2038 & $-0.6118 *$ & -0.0316 & 0.0551 & 0.0870 & 0.2831 & 0.2689 \\
\hline T. longibrachiatum & 0.2075 & -0.2341 & 0.4368 & 0.2745 & -0.0114 & 0.0965 & -0.0523 \\
\hline T. ressei & -0.1976 & 0.2949 & -0.0296 & -0.1212 & 0.0387 & -0.2240 & -0.4453 \\
\hline
\end{tabular}


Fig.1 Prevalence of bio-agents and phytopathogens in Bundelkhand region of U.P.

\section{Prevalence in Bundelkhand Region}
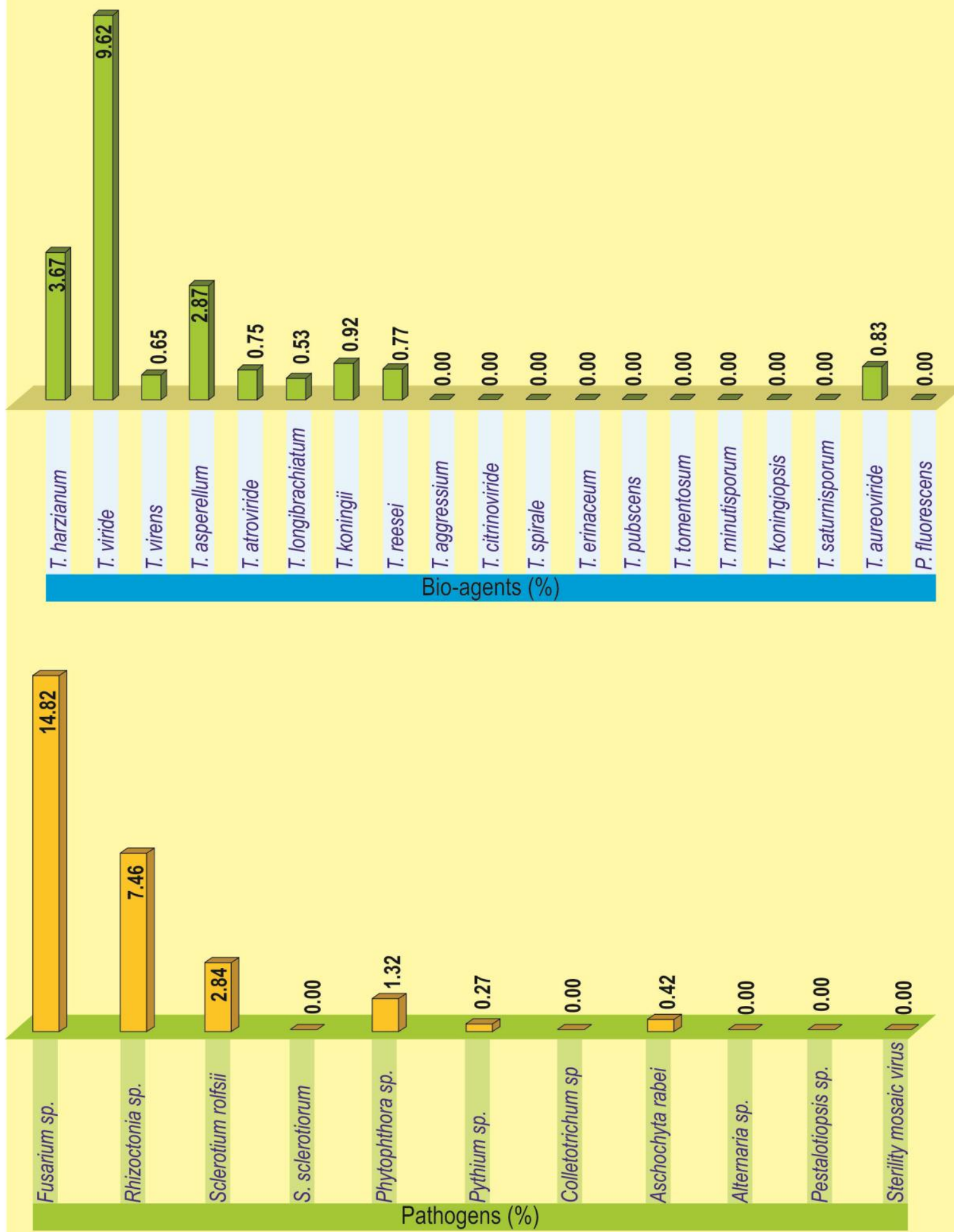
Fig.2 Prevalence of bio-agents and phytopathogens in Central region of U.P.

\section{Prevalence in Central Region}
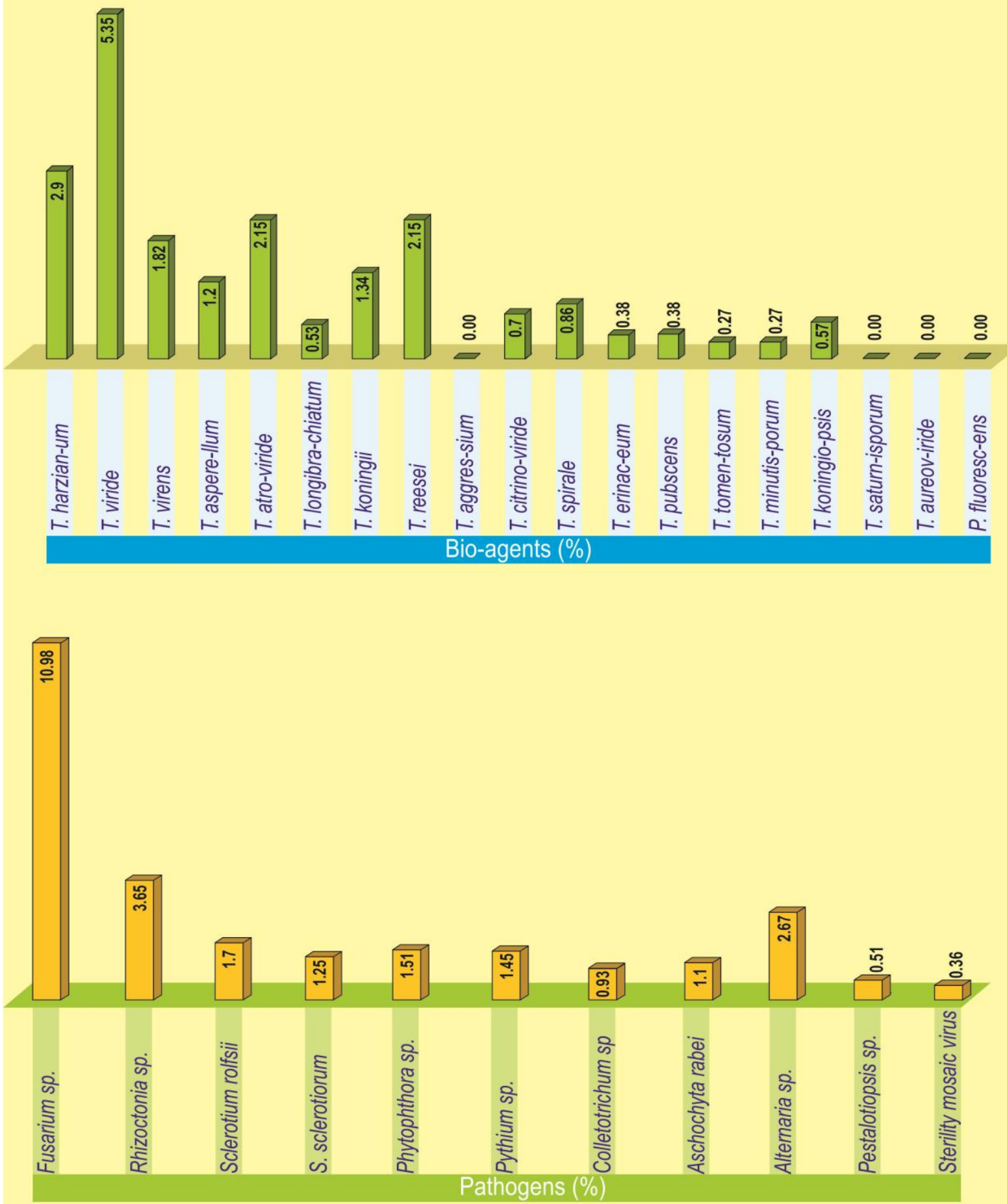
Fig.3 Prevalence of bio-agents and phytopathogens in Eastern region of U.P.

\section{Prevalence in Eastern Region}
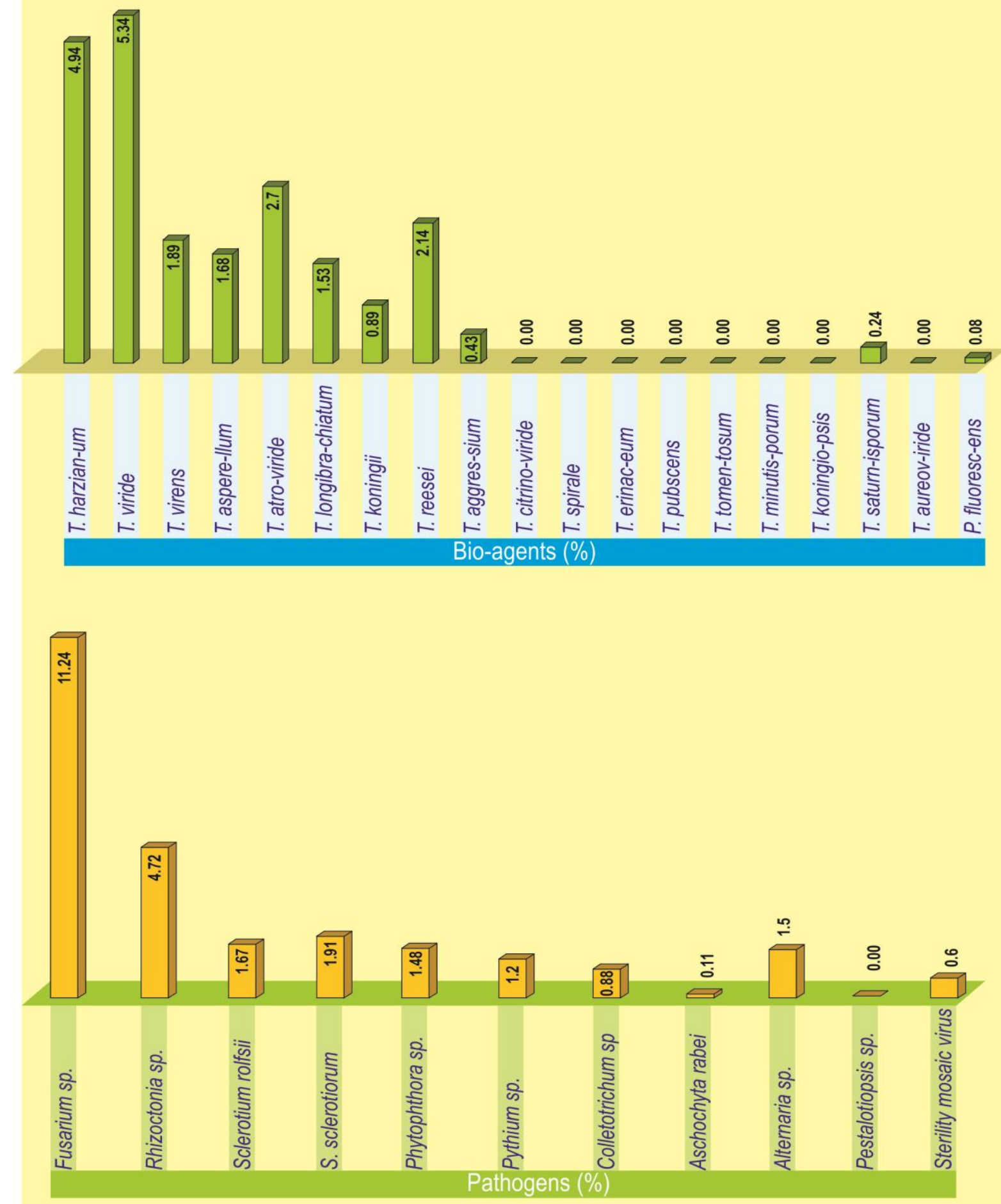
Fig.4 Average prevalence of bioagents and phytopathogens in three agroclimatic zones of U.P.

\section{Average Prevalence of Bio-agents}

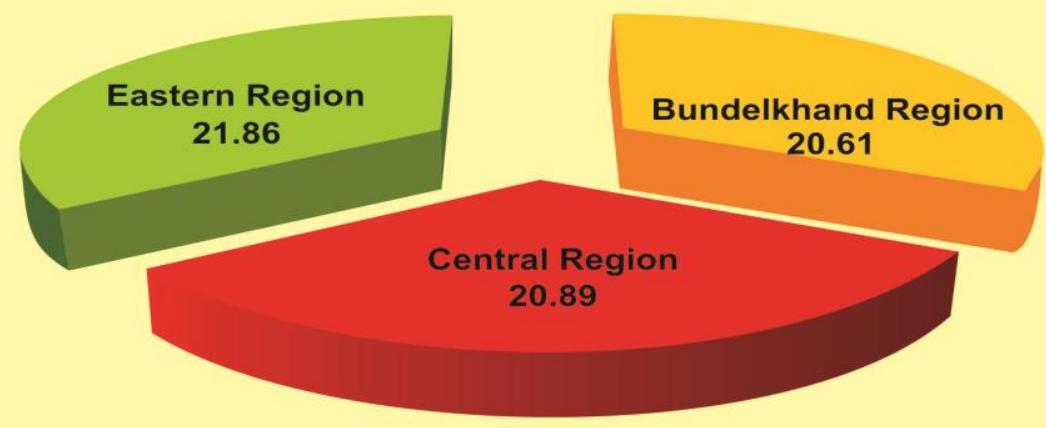

\section{Average Prevalence of Pathogens}

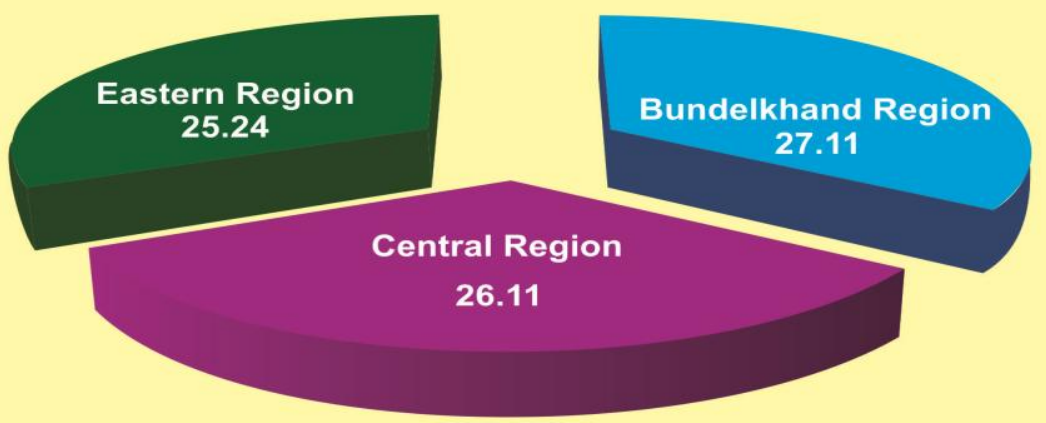

T. viride has given most effective response to the pathogens while, $T$. atroviride, T. reesei, T. harzianum. T. virens and T. asperellum were given statistically same response at 5 per cent level of significance in Auraiya, Kanpur Nagar, Kanpur Dehat and Etawah.

In addition, eleven species of bioagents viz; $T$. harzianum, T. viride, T. virens, T. asperellum, $T$. atroviride, $T$. longibrachiatum, T. koningii,
T. ressei, T. aggrissivum, T. saturnisporum and Pseudomonas fluorescens could be isolated from Eastern region of U.P. 9 species of bioagents from 9 Blocks of Allahabad; 8 species of bioagents from 6 Blocks of Sonbhadra, 7 species of bioagents from 9 Blocks of Azamgarh, 8 Blocks of Barabanki, 9 Blocks of Jaunpur, 6 Blocks of Mirzapur and 5 Blocks of Pratapgarh; 6 species of bioagents from 6 Blocks of Faizabad, 5 
Blocks each of Gonda, Sant Ravi Das Nagar and 9 Blocks of Sultanpur; 5 species of bioagents from 5 Blocks each of Bahraich, Ghazipur and 6 Blocks of Varanasi. In general, 21.86 per cent bioagents were isolated from this zone.

$T$. viride and $T$. harzianum were recorded statistically at par at 5 per cent level of significance given same response towards pathogens in Mirzapur, Jaunpur, Allahabad, Bahraich and Sultanpur. T. atroviride, $T$. reesei, $T$. virens, $T$. asperellum and $T$. longibrachiatum were also found effective in Allahabad, Mirzapur, Jaunpur, Pratapgarh and Sant Ravidas Nagar (Fig. 1, 2 \& 3).

These findings are in accordance with the findings of Tewari and Mukhopadhyay, 2001; Momol and Pernezny, 2005; Sariah et al., 2005; Kumar et al., 2012; Hilda et al., 2012.

\section{Analysis of correlation between pathogen and bioagent}

In case of zone wise co-efficient correlation between pathogen and bioagent it was found that in Bundelkhand region, Fusarium sp. were affected by $T$. harzianum and $T$. asperellum; $S$. rolfsii with $T$. harzianum and T. koningii and Phytophthora with T. viride, $T$. asperellum and $T$. koningii. In Central region, Fusarium sp. were affected by $T$. atroviride, Rhizoctonia sp. by $T$. harzianum, $T$. virens, $T$. asperellum and $T$. ressei. Similarly, $S$. rolfsii by $T$. harzianum, $T$. viride, $T$. atroviride and T. ressei, Sclerotina by $T$. harzianum, Phytophthora by $T$. virens, $T$. asperellum, T. atroviride, T. koningii and $T$. ressei, Pythium by $T$. viride, T. asperellum, $T$. atroviride, $T$. koningii and $T$ ressei, Colletotrichum sp. by $T$. harzianum and $T$. asperellum, Ascochyta rabei by $T$. harzianum and $T$. atroviride and Alternaria sp. by $T$. harzianum, $T$. viride, $T$. virens and $T$. asperellum. It was also observed that $T$. harzianum showed negative correlation with as many as 6 fungal pathogens.

In Eastern region, Fusarium sp. was affected by $T$. harzianum, $T$. virens and $T$. ressei; Rhizoctonia sp. by $T$. viride, T. asperellum, $T$. atroviride and $T$. ressei; $S$. rolfsii by $T$. harzianum, T. virens, T. atroviride and $T$ ressei; Sclerotina sp. by $T$. virens and $T$. ressei; Phytophthora sp. by $T$. harzianum, $T$. asperellum and T. longibrachiatum; Pythium sp. by $T$. harzianum and $T$ ressei; Alternaria sp. by $T$. harzianum, $T$. viride, $T$. asperellum, $T$. longibrachiatum and T. ressei. It was also observed that $T$. harzianum and $T$. ressei showed negative correlation with as many as 5 fungal pathogens.

Thereby these bioagents are responsible for reduction in overall population of the pathogens in this region (Table 3, Fig. 4).

From the survey made during 2011-12 \& 2012-13, it was concluded that the fields having much prevalence of bioagents showed low infestation of wilt/ root rot diseases. Based on the survey conducted in 203 blocks of 31 districts of 3 agro-climatic regions, the bioagents like Trichoderma viride and Trichoderma harzianum were prevalent in most part of the survey area of Uttar Pradesh. Other bioagents in terms of prevalence were $T$. atroviride, $T$. ressei, $T$. asperellum, $T$. virens, $T$. longibrachiatum, T. koningii, $T$. citrinoviride, T. koningiopsis, T. aggressivum, T. aureoviride, T. erinaceum, T. pubscens, $T$. saturnisporum, T. tomentosum, $T$. minutisporum and T. spirale.

Based on the survey, it was found that majority of soil borne pathogens are responsible for low yield of the pulse crops. Accidently, it was interesting to note that these soil micro-floras do harbour the bioagents, which can reduce the incidence of the disease largely. 


\section{Acknowledgments}

The authors are grateful to Department of Science and Technology New Delhi, (SR/WOS-A/LS-1182/2014) and Indian Council of Agricultural Research (ICAR), for providing necessary facilities to carried out the experiment.

\section{References}

Barnett HL, Hunter BB. 1972. Illustrated genera of imperfect fungi. Burgess Publishing Company. Minneapolis, Minnesota, $241 \mathrm{pp}$.

Chet 1987, Chet I. 1987. Trichoderma application, mode of action, and potential as a biocontrol agent of soilborne plant pathogenic fungi. In: I. Chet (ed.), Innovative Approaches to Plant Disease Control, pp. 137-160. John Wiley \& Sons: New York.

Chet I, Harman GE, Baker R. 1981. Trichoderma hamatum: its hyphal interactions with Rhizoctonia solani and Pythium spp. Microbial Ecology. 7, 29-38.

Elad YI, Chet Henis Y. 1991. A selective medium for improving quantitative isolation of Trichoderma spp. from soil. Phytoparasitica. 9: 59-67.

Elizabeth M, Lamb N, Erin F, Rosskop, Ronald Sonoda M. 2001. Fusarium Stem Rot of Greenhouse Peppers. In "Horticultural Sciences Department, Florida Cooperative Extension Service, Institute of Food and Agricultural Sciences, University of Florida. 'http://edis.ifas.ufl.edu’.

FAOSTAT. 2014. FAOSTAT - Statistical Database, 2014.

Gilman JC. 1957. A manual of soil fungi. Iowa State University Press, Ames, Iowa, U.S.A. 450pp.

Gurha SN, Chaudhary RG, Trivedi S. 2007. Root pathogens of chickpea in eastern and central Uttar Pradesh. Paper presented in National Symposium on "Legumes for ecological sustainability: Emerging challenges and opportunities" organized by Indian Society of Pulses research \& Development, November 35 pp-157.

Hilda G, García-Núñez, Sergio de J, RomeroGómez, Carlos E González-Esquivel, E. Gabino Nava-Bernal, A. Roberto Martínez-Campos. 2012. Isolation of native strains of Trichoderma spp, from horticultural soils of the valley of toluca, for potential biocontrol of sclerotinia. Tropical and Subtropical Agroecosystems, 15:357-365.

Johnson LA. 1957. Effect of antibiotics on the number of bacteria and fungi isolated and fungi isolated from soil by dilution plate method. Phytopathogy. 47: 21-22.

Jones PH. 2001. Preconstruction Considerations - Florida Greenhouse Vegetable Production Handbook, the Florida Greenhouse Vegetable Production Handbook. Florida Cooperative Extension Service, Institute of Food and Agricultural Sciences, University of Florida. at 'http://edis.ifas.ufl.edu'

Jude BT, Richard AA. 2001. Northeast Pepper Integrated Pest Management (IPM) Manual. University of Connecticut, Cooperative Extension System. pp: 136.

Kumar and Mukharjii. 1996. Kumar RN and Mukerji KG, 1996. Integrated disease management futureperspectives, $\mathrm{pp}$. 335-347. In: K.G. Mukerji, B. Mathur, B.P. Chamala and C. Chitralekha (Eds.), Advances in Botany. APH Publishing Corporation, New Delhi.

Kumar K, Amaresan N, Bhagat S, Madhuri K, Srivastava RC. 2012. Isolation and characterization of Trichoderma spp. for antagonistic activity against root rot and foliar pathogens. Indian $\mathbf{J}$ 
Microbiol. 52(2): 137-144.

Momol T, Pernezny K. 2005. Florida Plant Disease Management Guide: Tomato. Plant Disease Management Guide, Florida Cooperative Extension Service, Institute of Food and Agricultural Sciences, University of Florida. At http://edis.ifas.ufl.edu`.

Nelson PE, Toussoun TA, Marasan WFO. 1983. Fusarium spp. An illustrated manual for identification. The Pennsylvania Univ. Press, press, park. $218 \mathrm{pp}$.

Ngo BH, Vu DN, Tran DQ. 2006. Analyze antagonist effects of Trichoderma spp. for controlling southern stem rot caused by Sclerotium rolfsii on peanut. Plant Prot. 1:12-14.

Papavizas GG. 1985. Trichoderma and Gliocladium: Biology, ecology and potential for biocontrol. Ann. Rev. Phytopathol. 23: 23 - 54.

Pernezny K, Roberts PD, Murphy JF, Goldberg NP. 2003. Compendium of Pepper Diseases. APS Press, St. Paul, MN, pp: 63.
Rini CR, Sulochana KK. 2007. Usefulness of Trichoderma and Pseudomonas against Rhizoctonia solani and Fusarium oxysporum infecting tomato. J. Trop. Agric. 45: 21-28.

Sariah M, Choo CW, Zakaria H, Norihan MS. $2005 . \quad$ Quantification and characterisation of Trichoderma spp. from different ecosystems. Mycopathologia.159 (1):113-7.

SAS Institute Inc., 1996, 'SAS/STAT user's guide. Version 6. Vol. 2.' 12th edn. (SAS Institute Inc.: Cary, NC) 846 pp.

Tewari AK, Mukhopadhyay AN. 2001. Testing of different formulations of Gliocladium virens against chickpea wilt complex. Indian Phytopath. 54: 6771.

Tran TT. 1998. Antagonistic effectiveness of Trichoderma against plant fungal pathogens. Plant Prot., 4: 35-38.

Trivedi S, Gurha SN. 2006. Status of some soil borne pathogen infecting chickpea (Cicer arietinum L.) in Bundelkhand region of Uttar Pradesh. Journal of Food Legumes. 19 (1): 88-90.

\section{How to cite this article:}

Shubha Trivedi, Mukesh Srivastava, A.K. Srivastava, Ved Ratan, Mo. Shahid, Anuradha Singh, Sonika Pandey, Supriya Dixit and Srivastava, Y.K. 2017. Status of Root and Foliar Fungal Diseases of Pulses at Different Agro-Climatic Zones of Uttar Pradesh, India. Int.J.Curr.Microbiol.App.Sci. 6(11): 152-165. doi: https://doi.org/10.20546/ijcmas.2017.611.020 